\title{
BURDEN OF THROMBOCYTOPENIA IN CRITICALLY ILL PATIENTS AT A TERTIARY CARE HOSPITAL
}

\author{
DR. FAIZA ANAM \\ MBBS, Nishtar Hospital, Multan, Pakistan. \\ DR. FATIMA BIBI \\ MBBS, Nishtar Hospital, Multan, Pakistan.
}

DR. SIDRA KHALID

MBBS, Nishtar Hospital, Multan, Pakistan.

\begin{abstract}
Background; Neonatal thrombocytopenia is one of the commonest hematological abnormality encountered in neonatal intensive care unit. Though thrombocytopenia is prevalent it is often overlooked assuming it will resolve spontaneously. However, if it is not detected and managed properly can result in devastating complications. Objective; To determine the frequency of thrombocytopenia in sick neonates admitted in neonatal intensive care unit (NICU). Material and Methods; A total 282 newborns admitted to NICU of department of Pediatrics, Nishtar Hospital, Multan were included in this cross-sectional were enrolled, their blood samples were taken for estimation of platelet count to diagnose thrombocytopenia and all the data was analyzed by SPSS. Results; Of these 282 study cases, $198(70.2 \%)$ were male patients while $84(29.8 \%)$ were female patients. Mean age of our study cases was $5.18 \pm 3.69$ days. Of these 282 study cases, 85 (30.1\%) belonged to rural areas and 197 (69.9\%) belonged to urban areas. Of these 282 study cases, $187(66.3 \%)$ mothers were illiterate while $95(33.3 \%)$ were literate. Preterm birth was noted in $116(41.1 \%)$ of our study cases. Of these 282 study cases, $188(66.7 \%)$ were born through vaginal deliveries while $94(33.3 \%)$ by cesarean section. Mean duration of disease was $2.34 \pm 1.10$ days and 241 $(85.5 \%)$ had disease duration up to 3 days. Mean platelet count of our study cases was $156575.24 \pm 50250.53 \mathrm{~mm}^{3}$ and thrombocytopenia was present in $104(36.9 \%)$. Conclusion; High frequency of thrombocytopenia was observed in sick neonates in our study. Thrombocytopenia was significantly associated with age, residential status, maternal literacy, preterm births, mode of delivery and disease duration. All the clinicians treating such patients should regularly monitor platelet count of all the patients admitted to NICU for early diagnosis and timely management to save them further complications. This will not only decrease disease morbidity but will also reduce mortalities in these patients.
\end{abstract}

Keywords; Thrombocytopenia, sick neonates, Frequency.

DOI: $10.7176 / \mathrm{JMPB} / 52-11$

\section{INTRODUCTION}

Thrombocytopenia is the most common hemostatic abnormality in sick newborn infants ${ }^{1-3}$. Although many conditions may be associated with neonatal thrombocytopenia, low platelet counts in the first few days of life are often caused by fetomaternal problems, whereas thrombocytopenia developing after the third day is usually secondary to sepsis or necrotizing enterocolitis. Despite the frequency of thrombocytopenia in sick neonates, the underlying kinetic mechanisms are not always clear. The commonly accepted theories of decreased platelet production or increased consumption are being scrutinized in the light of new evidence. Thrombopoietin and interleukin-11 are being investigated to unravel the complex pathophysiology of thrombocytopenia in neonates 
and they are also being explored as potential therapeutic agents. Guidelines for platelet transfusions continue to be variable and controversial.

Thrombocytopenia is common among sick neonates, affecting $20-35 \%$ of all patients admitted to the neonatal intensive care unit (NICU) $)^{4-6}$. While most cases of neonatal thrombocytopenia (NT) are mild or moderate and resolve within 7-14 days with appropriate therapy, $2.5-5 \%$ of NICU patients develop severe thrombocytopenia, sometimes lasting for several weeks and requiring $>20$ platelet transfusions ${ }^{7,8}$.

Early onset thrombocytopenia is commonly associated with pregnancy complications such as intrauterine growth restriction, HELLP syndrome (hemolysis, elevated liver enzymes, and low platelet count), maternal diabetes or drug use. Clinically, the most common cause of severe early NT is known as neonatal alloimmune thrombocytopenia purpura (NAITP). However, NAITP accounts for only a small proportion $(<5 \%)$ of early NT overall. NT occurs in a large proportion of preterm infants, although the thrombocytopenia is self-limiting. It usually disappeares within 10 days ${ }^{9}$. In a study done at Lahore thrombocytopenia was present in $24.1 \%$ of the sick neonates admitted at Neonatal ICU (NICU) ${ }^{10}$.

The aim of my study is to determine the frequency of thrombocytopenia in sick neonates

\section{MATERIAL AND METHODS;}

A total of 282 sick neonates admitted to NICU of department of Pediatrics, Nishtar Hospital, Multan were included in this cross-sectional study. Patients who took platelet transfusion, with congenital heart diseases (like VSD, ASD and PDA) and bleeding disorders (Like hemophilia) were excluded from our study. Once registered in the study, all the relevant baseline investigations were done. Venous blood sample $(5 \mathrm{ml})$ was drawn and sent to laboratory of hospital for estimation of thrombocytopenia. All the data was entered and analyzed using SPSS-18.

\section{RESULTS}

Our study comprised of a total of 282 patients meeting inclusion criteria of our study. Of these 282 study cases, $198(70.2 \%)$ were male patients while $84(29.8 \%)$ were female patients. Mean age of our study cases was $5.18 \pm$ 3.69 days (with minimum age of our study cases was 1 day while maximum age was 16 days). Mean age of the male patients was noted to be $4.80 \pm 3.08$ days while that female patients was $6.10 \pm 4.72$ days $(p=0.007)$. Our study results have indicated that majority of our study cases i.e. $261(92.6 \%)$ were aged up to 15 days. Of these 282 study cases, $85(30.1 \%)$ belonged to rural areas and $197(69.9 \%)$ belonged to urban areas. Of these 282 study cases, 187 (66.3\%) mothers were illiterate while 95 (33.3\%) were literate. Preterm birth was noted in 116 (41.1 $\%)$ of our study cases. Of these 282 study cases, $188(66.7 \%)$ were born through vaginal deliveries while 94 $(33.3 \%)$ by cesarean section. Mean duration of disease was $2.34 \pm 1.10$ days and $241(85.5 \%)$ had disease duration up to 3 days. Mean platelet count of our study cases was $156575.24 \pm 50250.53 \mathrm{~mm}^{3}$ and thrombocytopenia was present in $104(36.9 \%)$.

\section{DISCUSSION}

Platelets are highly organized anuclear cellular fragments involved in primary hemostasis. Megakaryocyte progenitor cells develop under the stimulus of thrombopoietin to produce platelets ${ }^{11,12}$. Mature megakaryocytes then generate and release platelets into the bloodstream, where they have a half-life of 7 to 10 days. Platelets act by attaching to adhesion molecules exposed by breaks in endothelial walls, aggregating together and altering their shape (primary hemostasis) ${ }^{13-15}$.

Our study comprised of a total of 282 patients meeting inclusion criteria of our study. Of these 282 study cases, $198(70.2 \%)$ were male patients while $84(29.8 \%)$ were female patients. A study conducted by Kiran et al ${ }^{16}$ from India has also reported high male gender predominance with $63 \%$ male patients which is in compliance with our study results. A study conducted by Sharma et al ${ }^{17}$ has also reported high male gender preponderance with $84 \%$ male patients which is close to our study results. 
Mean age of our study cases was $5.18 \pm 3.69$ days (with minimum age of our study cases was 1 day while maximum age was 16 days). Mean age of the male patients was noted to be $4.80 \pm 3.08$ days while that female patients was $6.10 \pm 4.72$ days $(p=0.007)$. Our study results have indicated that majority of our study cases i.e. $261(92.6 \%)$ were aged up to 15 days. A study conducted by Kiran et al ${ }^{16}$ from India has also reported mean age was $92.86 \pm$ 143.75 hours which is in compliance with our study results.

Of these 282 study cases, 85 (30.1\%) belonged to rural areas and $197(69.9 \%)$ belonged to urban areas. Of these 282 study cases, $187(66.3 \%)$ mothers were illiterate while $95(33.3 \%)$ were literate. Preterm birth was noted in $116(41.1 \%)$ of our study cases. A study conducted by Kiran et al ${ }^{16}$ from India has also reported $43.8 \%$ prematurity which is close to our study results. A study conducted by Sharma et al ${ }^{17}$ has also reported $55 \%$ prematurity which is slightly higher than our study results.

Mean duration of disease was $2.34 \pm 1.10$ days and $241(85.5 \%)$ had disease duration up to 3 days. Mean platelet count of our study cases was $156575.24 \pm 50250.53 \mathrm{~mm}^{3}$ and thrombocytopenia was present in 104 (36.9\%). A study conducted by Kiran et al ${ }^{16}$ from India has also reported $35.30 \%$ thrombocytopenia in sick neonates which is close to our study results. A study conducted by Sharma et al ${ }^{17}$ has also reported $55 \%$ thrombocytopenia which is higher than our study results. A study conducted by Nandyal et al ${ }^{18}$ has also documented $63.8 \%$ thrombocytopenia in sick neonates which is higher than our findings.

\section{CONCLUSION}

High frequency of thrombocytopenia was observed in sick neonates in our study. Thrombocytopenia was significantly associated with age, residential status, maternal literacy, preterm births, mode of delivery and disease duration. All the clinicians treating such patients should regularly monitor platelet count of all the patients admitted to NICU for early diagnosis and timely management to save them further complications. This will not only decrease disease morbidity but will also reduce mortalities in these patients.

\section{REFERENCES}

1. Arif $\mathrm{SH}^{1}$, Ahmad I, Ali SM, Khan HM. Thrombocytopenia and bacterial sepsis in neonates. Indian J Hematol Blood Transfus. 2012 Sep;28(3):147-51.

2. Eissa DS ${ }^{1}$, El-Farrash RA. New insights into thrombopoiesis in neonatal sepsis. Platelets. 2013;24(2):122-8.

3. Gupta $\mathrm{AK}^{1}$, Kumari S, Singhal A, Bahl A. Neonatal thrombocytopenia and platelets transfusion. Asian J Transfus Sci. 2012 Jul;6(2):161-4.

4. Del Vecchio $\mathrm{A}^{1}$. Evaluation and management of thrombocytopenic neonates in the intensive care unit. Early Hum Dev. 2014 Sep;90 Suppl 2:S51-5.

5. Gupta $\mathrm{AK}^{1}$, Kumari S, Singhal A, Bahl A. Neonatal thrombocytopenia and platelets transfusion. Asian J Transfus Sci. 2012 Jul;6(2):161-4.

6. Christensen $\mathrm{RD}^{1}$, Henry E, Del Vecchio A. Thrombocytosis and thrombocytopenia in the NICU: incidence, mechanisms and treatments. J Matern Fetal Neonatal Med. 2012 Oct;25 Suppl 4:15-7.

7. Ferrer-Marin $\mathrm{F}^{1}$, Liu ZJ, Gutti R, Sola-Visner M. Neonatal thrombocytopenia and megakaryocytopoiesis. Semin Hematol. 2010 Jul;47(3):281-8.

8. Kandi R ${ }^{1}$, Gutti U, Undi R, Sahu I, Gutti RK. Understanding thrombocytopenia: physiological role of microRNA in survival of neonatal megakaryocytes. J Thromb Thrombolysis. 2015 Oct;40(3):310-6.

9. Eslami $\mathrm{Z}^{1}$, Lookzadeh $\mathrm{MH}^{2}$, Noorishadkam $\mathrm{M}^{1}$, Hashemi $\mathrm{A}^{1}$, Ghilian $\mathrm{R}^{3}$, Pirdehghan $\mathrm{A}^{4}$. Thrombocytopenia and associated factors in neonates admitted to NICU during Years 2010_2011. Iran J Ped Hematol Oncol. 2013;3(1):205-15.

10. Aman $\mathrm{I}^{1}$, Hassan KA, Ahmad TM. The study of thrombocytopenia in sick neonates. J Coll Physicians Surg Pak. 2004 May;14(5):282-5.

11. Ghevaert C, Campbell K, Walton J, Smith GA, Allen D, Williamson LM, et al. Management and outcome of 200 cases of fetomaternal alloimmune thrombocytopenia. Transfusion. 2007;47:901-10. 
12. Honohan A, van't Ende E, Hulzebos C, Lopriore E, van't Verlaat E, Govaert P, et al. Posttransfusion platelet increments after different platelet products in neonates: a retrospective cohort study. Transfusion. 2013 Dec;53(12):3100-9.

13. Han KH, Lee H, Kang HG, Moon KC, Lee JH, Park YS, et al. Renal manifestations of patients with MYH9-related disorders. Pediatr Nephrol. 2011;26:549-55.

14. Balduini CL, De Candia E, Savoia A. Why the disorder induced by GATA1 Arg216Gln mutation should be called "X-linked thrombocytopenia with thalassemia" rather than "X-linked gray platelet syndrome". Blood. 2007;110:2770-1.

15. Sillers L, Van Slambrouck C, Lapping-Carr G. Neonatal Thrombocytopenia: Etiology and Diagnosis. Pediatr Ann. 2015 Jul;44(7):e175-80. doi: 10.3928/00904481-20150710-11.

16. Kiran S, Bhaskar G, Jayashree N. A study of thrombocytopenia in sick neonates at a tertiary care hospital. Scholars J Applied Med Sic. 2017;5(9):3609-16.

17. Sharma A, Thapar K. A prospective observational study of thrombocytopenia in high risk neonates in a tertiary care teaching hospital. Sri J Child Health. 2015;44(4):213-19.

18. Nandyal SS, Shashikala P, Sahgal V. Study of thrombocytopenia in neonatal intensive care unit. Indian J Pathol Oncol. 2016;3(1):55-9. 